\title{
Introducción al cálculo particular y la dinámica de universos discretizables
}

Fredy Vides*

\section{RESUMEN}

El propósito del presente artículo es presentar los principios elementales sobre los que descansa la herramienta teórica a la que hemos nombrado Análisis Particular.

El Análisis Particular es un esfuerzo por extender el aparato que en general descansa sobre las ideas de Newton, Leibnitz, Fourier y otros más, a terrenos en los cuales suele presentar problemas debido a las exigencias directas sobre los atributos de los entes representativos (funciones) en un sistema discretizable.

Palabras Clave: Cálculo Particular, Universos Discretizables, Funciones de Ligadura, Representaciones Relativas, Principio de Equivalencia, Hermítico, Puntos de Equilibrio.

\section{ABSTRACT}

The purpose of the present article is to present the main principles who are the basis of the theoretical tool the we've namely Particular Analysis.

The Particular Analysis is an effort to extend the conventional approach who's based on Newton, Leibntiz and Fourier and others ideas, to applications where use to present some problems due to direct exigencies of the representative entities (functions) in a discretizable system.

Keywords: Particular Calculus, Discretizable Universes, String Functions, Relative Representations, Equivalence Principle, Hermitic, Equilibrium Points.

\footnotetext{
*Fredy Vides, fredyvides@gmail.com

Facultad de Ciencias, Departamento de Matemática. Universidad Nacional Autónoma de Honduras
} 


\section{INTRODUCCIÓN}

El propósito del presente artículo es exponer el formalismo y correspondiente alcance característico del análisis particular.

Pretende ademas servir como base para futuras exposiciones y estudios referentes al tema, tanto para quienes deseen investigarlo o para quienes solamente busquen aplicarlo.

\section{PRELIMINARES}

El primer concepto a desarrollar dentro del análisis particular es el significado de ente representativo (función). Esencialmente tendremos 2 tipos de funciones, clasificadas según las siguientes condiciones:

\section{Funciones particulares monoligadas:}

Funciones cuya definición viene dada de la siguiente manera:

Siendo $X_{p}$ un conjunto que recibe el nombre de sistema de coordenadas particulares y que viene definido de la forma

$$
X_{p}=X_{p}^{m}=\left\{x_{i} \in \mathbb{R}^{m}: x_{i}=\mathcal{H}(i ; l), i \in \mathfrak{J} \subset \mathbb{Z}^{+}\right\}
$$

en donde $H(i ; I)=h(i, I) e_{j}, 1 j m$; una función monoligada $f$ es aquella que puede ser abreviada en la forma:

$$
\hat{f}=\hat{f}_{m}^{p}(t ; k)=\left(F_{p}^{m}, \alpha\right)
$$

Con

$$
F_{p}^{m}=\left\{y_{i} \in \mathbb{R}^{m}: y_{i}=f\left(x_{i}\right), x_{i} \in X_{p}\right\}
$$

En donde f recibe el nombre de función generadora y $\alpha_{k}: \underline{R} \supset J_{\mapsto} \mapsto \underline{\boldsymbol{R}}$ en función paramétrica de ligadura continua a trozos sujeta solamente a las siguientes condiciones.

$$
\begin{gathered}
\left\{\begin{array}{l}
\alpha(0 ; 1)=y_{1} \\
\alpha(1 ; i)=\alpha(0 ; i+1)=y_{i+1}, 1 \leqslant i \leq p-2 \\
\alpha(1 ; p-1)=y_{p}
\end{array}\right. \\
\alpha(t ; 0)=\{\} \\
\alpha(t ; k)=\{\}, k \geqslant p
\end{gathered}
$$


Las funciones monoligadas son apropiadas para el análisis particular aplicado a dinámica de sistemas de partículas representativas bajo la influencia de campos de fuerza conservativos.

\section{Funciones particulares multiligadas:}

Las funciones multiligadas se definen por:

$$
\tilde{f}=\tilde{f}_{m}^{p}(t ; k)=\left(F_{p}^{m}, \mathcal{A}\right)
$$

y difieren de las monoligadas en que aparte de que sus funciones de ligadura cumplen con las condiciones (1.1) satisfacen además condiciones adicionales que tienen que ver con las propiedades físicas del sistema, una de las condiciones frecuentes es satisfacer la ecuación:

$$
\left[D^{4}-\tau^{2} D^{2}\right] \mathcal{A}=0
$$

condición que junto a condiciones de suavidad en los nudos de $F_{p}$ se utiliza para obtener ciertos niveles de tensión sobre los nudos controlados por el parámetro t y acordes a las fuerzas que actúan en el sistema de partículas representativas.

\section{El espacio $E^{p, m}{ }_{f}$ de representaciones relativas:}

El conjunto de todas las funciones bien monoligadas o multiligadas, representa cada uno, un espacio tensorial de orden $p \times m$ definido en la forma:

$$
E_{f}^{p, m}=\hat{E}_{f}^{p, m}=\hat{E}_{f}=(\mathbb{C}, \hat{f}, \oplus, \odot)
$$

para funciones monoligadas 0

$$
E_{f}^{p, m}=\tilde{E}_{f}^{p, m}=\hat{E}_{f}=(\mathbb{C}, \hat{f}, \oplus, \odot)
$$

para funciones multiligadas.

La forma $\pi_{f, m}^{p, m} \cdot f$ se conoce como representación particular de $f$ relativa a $E_{f}^{p, m}$ y viene determinada por:

$$
\pi_{f}^{p, m} \cdot f=F_{p}^{m} \cup\left(\bigcup_{i \in \mathfrak{J}} \alpha(T ; i)\right), T=[0,1]
$$


En esta relación $\pi^{p, m}{ }_{f}$ recibe el nombre de operador de proyección hacia $E^{p, m}$.

\section{Descomposición proyectiva discreta:}

Al tener el espacio particular discreto inducido por una función bien mono 0 multiligada, que puede ser expresado en la forma:

$$
F_{f}^{p, m}=\hat{F}_{f}=\left(\mathbb{C}, F_{p}^{m}, \oplus, \odot\right)
$$

para funciones monoligadas $y$

$$
F_{f}^{p, m}=\tilde{F}_{f}=\left(\mathbb{C}, F_{p}^{m}, \oplus, \odot\right)
$$

para funciones multiligadas, puedea.C. notarse que

$$
\hat{F}_{f}=\tilde{F}_{f}
$$

No obstante surge entonces la necesidad de definir la familia base de subespacios a utilizar cuando realicemos procedimientos de definición de operadores y otras tareas propias del análisis particular de un sistema discretizable determinado por $\mathrm{F}_{\mathrm{f}, \mathrm{m}}$, que por simplicidad llamaremos $\mathrm{F}_{\mathrm{f}}$.

Para obtener dicha familia nos valdremos del operador de proyección simple, el cual vendrá definido en nuestro aparato como:

$$
\pi_{X}: F_{f} \mapsto X \subseteq F_{f}
$$

decimos que $\pi_{x}$ genera una familia base de subespacios en $F_{f}$ dado que

$$
\begin{aligned}
& F_{f}=\bigcup_{X \in \mathcal{P}\left(F_{f}\right)} \pi_{X} F_{f} \\
& \emptyset=\bigcap_{X \in \mathcal{P}\left(F_{f}\right)} \pi_{X} F_{f}
\end{aligned}
$$

En donde $P\left(F_{f}\right)$ es el conjunto de las partes de $F_{f}$ que satisfacen la relación anterior, por otro lado la forma en que construimos $F_{p}^{m}$ hace posible que $\pi_{x}$ pueda reemplazase cuando sea conveniente por $\pi_{i}$ que viene determinado por

$$
\pi_{i}=\pi_{F_{p}^{m, i}}^{m}
$$

en donde $\mathrm{F}_{p}^{\mathrm{m}, \mathrm{i}}$ es un conjunto que viene determinado por la relación:

$$
F_{p}^{m, i}=\left\{y: y=F_{p}^{m} \odot \Delta_{i}^{m}\right\}, 1 \leqslant i \leqslant m, p \in \mathcal{J}
$$


Con:

$$
\left(\Delta_{i}^{m}\right)_{k, l}=\delta_{i}^{k} \delta_{i}^{l}, 1 \leqslant k, l \leqslant m
$$

con base en esta redefinición puede verse que:

y por lo tanto:

$$
\pi_{i} \cdot(\cdot) \equiv(\cdot) \odot \Delta_{i}^{m}
$$

$$
F_{p}^{m}=\bigoplus_{1 \leqslant i \leqslant m} F_{p}^{m, i}
$$

\section{Forma particular de operadores:}

La forma particular de cualquier operador Hermitico en el entorno del análisis de un sistema dado viene inducida por el siguiente procedimiento:

Siendo $T$ el operador a representar y siendo $X_{p}$ el sistema de coordenadas particulares junto con $U$ un elemento fijo pero arbitrario en $F_{p}$, luego la representación particular de dicho operador vendrá determinada de la forma:

$$
\left[a_{t} a_{s} \tilde{T} \tilde{S}-\alpha\left[\pi_{F_{p}^{m}} \cdot T\right]\right] v=\pi_{f}^{p, m} \cdot r
$$

Si se tiene que Tes hermítico entonces el procedimiento anterior se puede expresar como:

$$
\left[a_{t}^{*} a_{t} \tilde{T}^{\star} \tilde{T}-\alpha\left[\pi_{F_{p}^{m}} \cdot T\right]\right] v=\pi_{f}^{p, m} \cdot r
$$

En donde $\alpha, a_{t} a_{s} \in \underline{\boldsymbol{C}}$ con escalares llamados escalares de ajuste y $\pi_{\chi_{p}}$ es el operador de proyección hacia $\mathrm{X}_{\mathrm{p}}$ cuyo papel consiste en recuperar la acción debida a la sub-distribución [2] de los elementos de una base de $\mathrm{F}_{\mathrm{p}}^{\mathrm{m}}$.

\section{Los operadores no hermíticos d e l y su definición:}

Dos operadores que serán de gran utilidad en nuestro estudio son el operador $\mathbf{d}$ que viene definido en el análisis particular de la siguiente manera:

$$
\text { d: } F_{p}^{m} \rightarrow F_{p}^{m}
$$

La cual es quizá una de las pocas ocasiones en que dicho operador aparece en forma explícita, dado que es un operador sumamente polimorfo, de modo que la 
forma en que viene representado en un conjunto $\mathrm{F}_{\mathrm{p}}^{\mathrm{m}}$ dependerá de la base funcional que prevalezca en dicho conjunto y además de la interacción con el operador I cuya definición esta dada por:

$$
\mathfrak{I}: \mathbb{C} \mapsto \mathcal{U} \subset \mathbb{R}
$$

el producto interno base en el análisis particular viene dado de la siguiente manera:

Siendo $\varphi, \psi \in F_{p}$ se define el producto interno en base en $\mathrm{F}_{\mathrm{p}}$ por:

$$
\langle\varphi \mid \psi\rangle=\mathfrak{I}(\varphi \circledast[\kappa \psi])
$$

Por otro lado si tenemos:

$$
\begin{array}{r}
\Psi: \mathbb{R}^{m} \supset \mathcal{W} \mapsto \mathbb{R}^{m} \\
\psi: \mathbb{R} \supset \mathcal{V} \mapsto \mathbb{R}
\end{array}
$$

los operadores d e l están además relacionados por las fórmulas:

$$
\begin{array}{r}
\mathfrak{I}\left(\pi_{i} \cdot(d \Psi)\right)=\mathfrak{I}\left(d \Psi^{i}\right)=\pi_{\mathcal{U}} \cdot(\mathcal{I} \Psi)=\mathcal{I}_{\mathcal{U}} \Psi_{\mathcal{U}} \\
d(\mathfrak{I}(\psi))=\mathcal{I}_{\mathcal{U}} \psi_{\mathcal{U}}
\end{array}
$$

\section{Principio de equivalencia de operadores en el análisis particular}

Dado un operador T, cuya acción es observada en un marco referencial:

$$
U_{\phi}^{f}=\left(E_{f}^{p}, m, \phi_{t}\right)
$$

Donde $\phi_{t}$ es un sistema dinámico polimorfo que resume la dinámica de este marco y esta a su vez directamente relacionado a las funciones de ligadura de $E_{\mathrm{f}, \mathrm{m}}^{\mathrm{p}}$. Un sistema multifísico observado en este marco que funciona bajo las leyes de Ty que sigue una evolución modelada por $\phi_{t}$ en la forma:

$$
\left(\phi_{t} \mathrm{o} T\right)\left(\psi_{s}\right)=\psi_{s+t}
$$

Endonde $s, t \in \underline{Z}$ y $\psi_{s}, \psi_{s+t} \in E_{f}^{p, m}$, se dice que es equivalente a:

$$
\left(\Phi_{\tau} \circ \tilde{T}\right)\left(\psi_{\sigma}^{\prime}\right)=\psi_{\sigma+\tau}^{\prime}
$$

Con

$$
U_{\Phi}^{g}=\left(E_{g}^{p, m}, \Phi_{\tau}\right)
$$

Siempre que: 


$$
\begin{array}{r}
\pi_{F_{p}^{m}} \cdot T u=f_{v} \\
\pi_{G_{p}^{m}} \cdot T v=g_{u} \\
\tilde{T} u=\alpha \hat{S}_{u} v+\epsilon H\left(f_{u}, g_{v}\right) \\
\tilde{T}^{\star} v=\beta \hat{S}_{v} u
\end{array}
$$

Yademás tanto $\phi_{t}$ como $\boldsymbol{\Phi}_{T}$ tienen los mismos puntos de equilibrio.

El sistema de ecuaciones (2) constituye la base del principio de equivalencia de operadores en el análisis particular. Otros aspectos importantes en el análisis particular son las equivalencias entre operadores por conversión de bases ortonormales sin embargo este tema corresponde a un escrito mas avanzado que el presente, si usted desea leer al respecto visite nuestro sitio web o envíenos un correo para que por esta vía podamos enviarle el artículo El Principio de Equivalencia en el Análisis Particular.

\section{APLICACIONES FÍSICAS}

Una de las propiedades mas importantes del análisis particular es que la estructura de sus funciones de operación en especial las multiligadas, permite que las mismas hereden propiedades físicas del marco en el que se esta trabajando que ayudan a que el modelo en el que se esta interesado presente un comportamiento tan ajustado a la realidad de su enfoque como se quiera.

\section{Ejemplo1:}

Dado el operador:

$$
L=-i \partial_{x}^{2}
$$

su forma particular discreta vendría dada en $\mathrm{E}^{\wedge_{\mathrm{p}, 1}}{ }_{\mathrm{f}}$ por:

$$
\left[c \hat{d}^{*} \hat{d}-\alpha\left[\pi_{F_{p}^{m}} \cdot\left(-i \partial_{x}^{2}\right)\right]\right] v=\pi_{f}^{p, m} \cdot r
$$

lo cual nos llevaría luego de resolver el sistema (2) a una representación de la forma:

$$
\hat{L} \equiv i \Im\left[\hat{d}_{p}^{\star} \hat{d}_{q}\right]
$$

\section{Ejemplo 2:}

Dado el operador:

$$
L=-i \partial_{x}^{2}+2 \partial_{x}
$$


Su forma particular discreta vendría dada en $E^{\Lambda_{p}, 1}$ por:

$$
\left[c \hat{d}^{t} \hat{d}^{s}-\alpha\left[\pi_{F_{p}^{m}} \cdot\left(-i \partial_{x}^{2}+2 \partial_{x}\right)\right]\right] v=\pi_{f}^{p, m} \cdot r
$$

lo cual nos llevaría luego de resolver el sistema (2) a una representación de la forma:

$$
\hat{L} \equiv i \mathfrak{I}\left[\hat{d}_{p}^{\star} \hat{d}_{q}\right]-2 \mathfrak{I}\left[\hat{d}_{p}^{\star} \mathcal{I}_{q}\right]
$$

\section{Ejemplo 3:}

Dado el operador:

$$
L=-i\left(\partial_{x}^{2}+\partial_{y}^{2}\right)
$$

su forma particular discreta vendría dada en $\mathrm{E}^{\wedge, 2}{ }_{\mathrm{f}}$ por:

$$
\left[c \hat{d}^{\star} \hat{d}-\alpha\left[\pi_{F_{p}^{m}} \cdot\left(-i\left(\partial_{x}^{2}+\partial_{y}^{2}\right)\right]\right] v=\pi_{f}^{p, m} \cdot r\right.
$$

lo cual nos llevaría luego de resolver el sistema (2) a una representación de la forma:

$$
\hat{L} \equiv i\left(\Im\left[\hat{d}_{p}^{\star} \hat{d}_{q}\right] \otimes \mathcal{I}_{2}+\mathcal{I}_{1} \otimes \mathfrak{I}\left[\hat{d}_{p}^{*} \hat{d}_{q}\right]\right)
$$

Además problemas no estacionarios de la física matemática pueden ser reinterpretados en el análisis particular.

\section{Ejemplo4:}

Dado el operador:

$$
L=\partial_{t}-i\left(\partial_{x}^{2}+\partial_{y}^{2}\right)
$$

su forma particular discreta vendría dada en $\mathrm{E}^{\wedge, 2}{ }_{\mathrm{f}}$ por:

$$
\left[c \hat{d}^{\star} \hat{d}-\alpha\left[\pi_{F_{p}^{m}} \cdot\left(-i\left(\partial_{x}^{2}+\partial_{y}^{2}\right)\right]\right] v=\pi_{f}^{p, m} \cdot r\right.
$$

lo cual nos llevaría luego de resolver el sistema (2) a una representación de la forma:

$$
\hat{L} \equiv i\left(\mathfrak{I}\left[\hat{d}_{p}^{*} \hat{d}_{q}\right] \otimes \mathcal{I}_{2}+\mathcal{I}_{1} \otimes \mathfrak{I}\left[\hat{d}_{p}^{*} \hat{d}_{q}\right]\right)
$$

luego la dinámica del sistema multifisico vendría dada por:

$$
\phi_{t} \circ \hat{L} \equiv \Im\left[\mathcal{I}_{p}\right]+h_{t} \hat{L}
$$




\section{CONCLUSIÓN}

El análisis particular es una herramienta teórica que pese a encontrarse en una fase temprana de desarrollo, logra plasmar de forma conveniente y precisa las leyes físicas que se observan en uno o más sistemas discretizables independientes 0 interrelacionados, de una manera especialmente satisfactoria, debido a que permite representar cualidades de los observables que de otro modo serían mas obscuras debido a que permite representar con excelente precisión procesos que de otro modo no podrían representarse debido a discontinuidades problemas de escala y cosas por el estilo.

\section{BIBLIOGRAFÍA}

F. Vides. Los Polinomios Ortogonales y las Matemáticas del Cálculo Científico. Revista de Ciencia y Tecnología. Noviembre 2002.

F. Vides. Fundamentos de Sub-Distribuciones. Boletín de Divulgación Interna del Depto. de Matemática. Noviembre 2002.

F. Vides. Modelado Numérico de Movimiento Ondulatorio en un Medio Heterogéneo No Isotrópico bajo Condiciones de Estabilidad Orbital. Boletín de Divulgación Interna del Depto. de Matemática. Julio 2006

S. Steinberg. A Discrete Calculus with Applications of High-Order Discretizations to Boundary-Value Problems. PASI/PANAM Honduras .October 5, 2004. 